\title{
A NOVEL APPROACH OF POWER-LINE MONITORING SYSTEM USING IOT
}

\section{SUDHARSHAN DUTH. P, ANIRUDH A. KODAGALI \& G. HARISH SINGH}

Department of Computer Science, Amrita School of Arts and Sciences, Amrita Vishwa Vidyapeetham, Mysuru, Karnataka, India

\begin{abstract}
The enhancement of internet of things along with its wide range of applications has made the future rich in technology and better controlling and monitoring of things remotely.

Post review of the current power monitoring system, it came to the notice that the manual controlling is still existing in large scale which has high risk involved including life threats. The probability of diagnosis of any issue in the power line and the fixation of the problem is time consuming and with less success rate. The proposed system make use of IOT which ensures high accuracy and provide intimations on detection of any issues in power line including heavy rainfall, fire due to short circuit along with the location of poles using its unique IDs where the issue has happened which helps to prevent or to reduce the impact. The data sent is uploaded in the cloud which contains all the IDs and compared for match followed by stopping of electric flow to the particular line along with broadcasting message to the authorities. The experimental analysis has proved high accuracy rate with a balanced computational time of the proposed system.

KEYWORDS-Internet of Things (IOT), Wireless Sensor Networks (WSN), ARDUINO UNO \& Sensors
\end{abstract}

Received: Apr 27, 2019; Accepted: May 18, 2019; Published: Jun 11, 2019; Paper Id.: IJMPERDJUN2019160

\section{INTRODUCTION}

In the current era of the technology IoT has proved its importance and efficiency. It is a network of sensors which communicates to each other through routers and makes life easier with ease of controlling and monitoring of devices. The connection between the devices has grown beyond the communications between humans and between human and devices. Each and every entity in the real world will be assigned with the IP address with the sense of regularising the usage and monitoring of issues regarding the object. Its application ranges from agriculture, health care, home appliances, smart city, smart grid and so on which helps to accomplish period dominant associate degree of correct management and to form an intelligent call of the physical world. The power line communications operate around the clock which demands more usage as the population increases.

This has not taken advantage of the technology to monitor the energy consumption and expenses. The issues regarding the power supply and the risks involved still persists. This also involves life threats, loss of assets and properties as a result of inaccurate monitoring of the system.

We make use of IOT technology to achieve the objective of our project, i. e., the power line monitoring system in which we can remotely sense the changes occurred in the $969 *$ power line due to natural calamities, power fluctuations, short circuit, etc. 


\section{LITERATURE SURVEY}

Ducpyo Hong, Jinmok Lee et al., (2005) this paper uses an approach on monitoring the PQ variation in a continuous manner with the help of PCL. From the experiments performed it is proved that PQ can be monitored from a remote place with the help of PCL. Without any interruption of new lines, it sends and receives the PQ information in a LAN network. The interruptions such as change in the Voltage and harmonic distortion causes malfunction in the equipment and increase the cost of production. So, they monitor all this power quality information at different remote places using LAN communication. Disadvantage: Powerline communication itself is a basic problem here if any wire is broken, the communication through Power lines won't takes place and the whole system is dead. Advantage in our project: In our project every sensors and node units assure exact power quality information and detects if any problem occurs like short circuit etc. and sends the data without any disturbance. Xi Chen, Limin Sun et al., (2012)In this paper a SG-IOT 3-layer architecture model is proposed. The effect of the natural disaster and the damage caused can be massively reduced by sending warning and by monitoring on a real time scale in case of disaster occurrence. With IOT human to machine and machine to machine exchange of data and uninterrupted fine knowledge flows. Electric power lines can be seen as the arteries of the modernized energy system where it has to be reliable, stable and efficient, as it can affect the nation's economic and political constraints in everyday life. The power line transmits high voltage that cross desserts, mountains and seas by transmitting a huge amount of electrical energy between both the regions and the sub-regions. More than the distribution equipment the transmission equipment's fail and cause power outages. The transmission halt affects more customers and leads much expensive outage costs. Due to this, it has gained a lot of attention on the transmission's reliability and stability. The overhead power lines are prone to be vulnerable to extreme weather conditions. Overhead high voltage transmission has common pitfalls such as wind deviation and wind vibrations. The conductor galloping that is caused due to strong winds lasted for several hours and brought great damage to the lines whereas the rainy and snowy weather causes frozen lines and also leaning of transmission lines will be caused due to the asymmetric pull which is a threat to the lines. The SG-IOT can be divided into three layers, first, the perception layer, second, the network layer and third, the application layer: the perception layer, the network layer and the application layer. The first layer consists of various kinds of sensors, multi-dimension code tags and readers, RFID tags and readers, cameras, WSN, GPS terminals, wired sensor networks, machine-to-machine (M2M) terminals, sensor gateways. The second layer is composed of the core networks and the telecommunication network. The third layer consists of the terminal units and infrastructure/middleware applications. Thus, this paper has complex task and is expensive too.

ZHOU Yao, WANG Wei et al., (2009) this paper shows that sensors that are built on the transmission line such that it reads the temperature change that occur in the real time world. This allows the base to analyze the dynamic increase in the capacity of transmission lines. From the result it is seen that reliable communication happens within $200 \mathrm{mts}$ of distance. On-Line Transmission is a temperature monitor system that consists of set of single base stations which include a wireless transceiver module and a micro-controller display unit, alarm unit, power modules and interface unit. Most of the commands are operated from the base stations, in return it will send those commands in Zigbee protocol, that has point-to-point protocol and realizes management of multi nodes. RS232 is used to connect the RS with the computer. The hardware and software requirements are real time data and less power consumption. From the results, it's known that for more than 200mts reliable communication takes place. Also, this has resolved the problem of outdoor environment conditions of reduced supply of power. It gives us an effective base for future scope on research of dynamic increase in capacity of Transmission lines. 
Qing Wang et al., (2012), the paper gives an approach to the system application that is based on the WIMAX and WIFI features, that is used for power line monitoring systems to increase wireless access. With the help of the results required by the simulation, it is seen that the performance of RER is degraded while it shares spectrum with others. Digital transmission was one of the means of communication for the power line monitoring system. Due to the disadvantages in digital and optical transmission they chose wireless transmission which included WIFI and WIMAX. Wireless transmission had distant advantages like maintenance, security and scalability. WIFI is used to transmit the sensed data in short range and WIMAX to transmit the sensed data to long range. They used $3 \mathrm{G}$ and $4 \mathrm{G}$ technologies to transmit the data. The proposed system says, the distance between the 2 sub stations is nearly $200 \mathrm{~km}$ and a base station is set at all the substations, so that every base station covers the radius of $100 \mathrm{~km}$.

\section{BACKGROUND}

\section{Arduino Uno}

Arduino is an open source tool where in which it consists of single board microcontroller kit for developing innovation things in the field of IoT. Using Arduino, we can customize things as per the user needs, for example, home automation etc.

\section{Arduino Uno REV3}

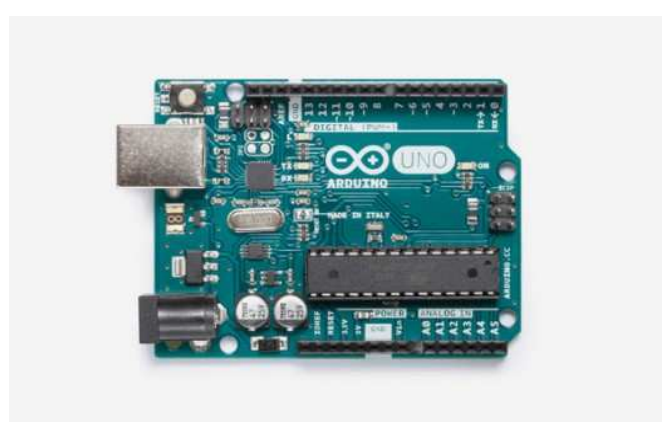

Figure 1: Arduino UNO REV3

Arduino board uses a wide range of microprocessor and controllers to control them. The board consists of analog and digital pins for input and output using which serial communications interface can be done and also universal serial bus on some model can be done. It uses $\mathrm{C}$ and $\mathrm{C}++$ for programming and it also provides IDE (Integrated Development Environment).

Arduino Uno is a microcontroller board with fourteen digital input output pins half of which is used as PWM outputs. It consists of everything that is required to support the microcontroller to connect to laptop with the help of USB cable or power it with an AC to DC adapter. In Arduino fourteen digital pins in the Uno is used for input and output and operates at 5 Volts each and every pin will offer $\mathrm{mA}$ as suggested. Usually $40 \mathrm{~mA}$ is worth to use as it contains control over input and output and it will avoid permanent harm to the microcontroller.

\section{ESP32}

ESP32 is a combination of microcontrollers embedded with WIFI and Bluetooth facility consisting of amplifier, filters and many more features. It has a memory of 520KiB SRAM and internal low-dropout regulator. It is owned by ESpressif Systems. The CPU used is Tensilica Xtensa LX6 microprocessor with $160 \mathrm{MHz}$ or $240 \mathrm{MHz}$. It provides with 
many security features which enhances its speciality like flash encryption, security boot and also cryptographic hardware acceleration.

The Sensors Used

Dht 11

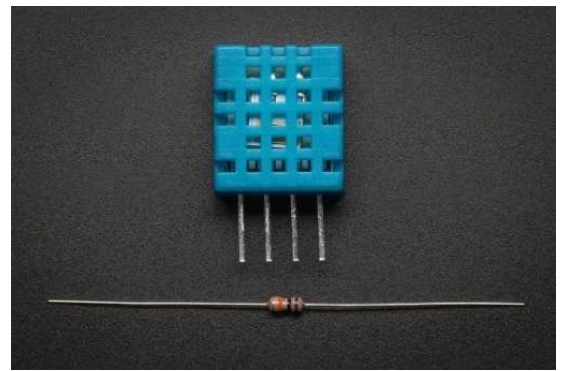

Figure 2: Dht11 Sensor

DHT11 is a basically a temperature and humidity sensor which uses a thermistor to calculate the air around it and gives a digital impulse on the pin which is used at every $20 \mathrm{sec}$ data is generated and can be compared with the existing recording.

\section{Fire Sensors}

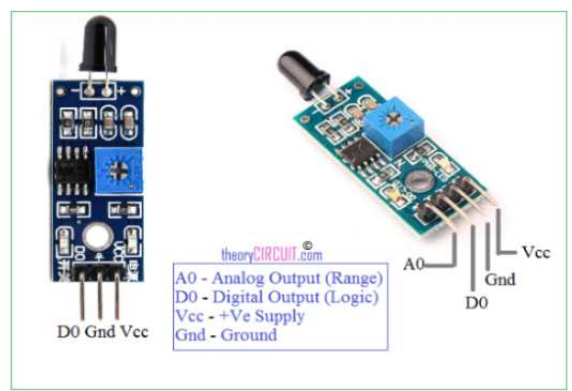

Figure 3: Fire Sensor

A fire sensor is sensing element that is desinged to detect the presence of a fire as soon as it detects the fire and sensor will start alerting by sounding an alarm, the sensor will detect the fire very quickly and alerts it. Usally this kind of sensors are used in industries and institutions etc. It is one of the cost effective sensor consisting of IR sensor using which it detects fire.

\section{Rain Sensor}

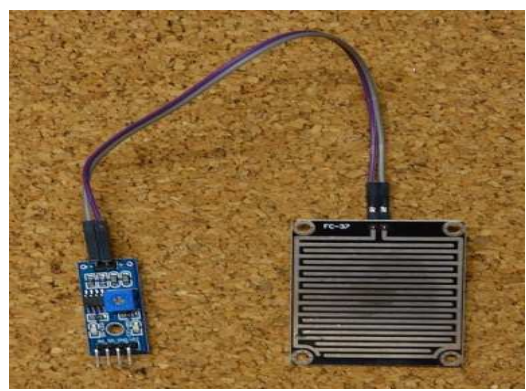

Figure 4: Rain Sensor 
The rain sensor is a simple tool for rain detection, when a rain drop is fall on the senor board's printed leads and it identifies it and the data is been calculated. It makes use of the variable resistor which changes when is wet and when it is dry. It has a wide range of applications mainly water conservation in agriculture and automated wipers.

\section{PROPOSED METHOD}

Flowing of Electricity can't be identified without any Instruments, and we can't see where the power line is broken. Many technologies are there to measure and monitor the power line, but we are here using a modern method to control and monitor the power line remotely. The power line holding poles are made of an insulator, and these are the basics support structures of a power grid.

The Isolated Node selection unit keeps on checking the poles by switching the Trans-receivers. Parallelly the short circuit and voltage/current monitor unit keep on checking the switching power line poles if Network. The website displays the status of the power line. Any variation found on the respective pole the unit will send a signal Microcontroller unit. The Microcontroller analyses the data and displays the current scenario on the display unit, the display unit displays the status of the poles on the LCD display for quick analyzation. By the same time of posting the status on an LCD, the Microcontroller will send an AT commands to WIFI module for transmitting the data to cloud data storage for remote data analysis on a website. The website is a is an open source for an Internet of Things (IoT) application and the API to store and to retrieve data using the HTTP protocol over the Internet or via a Local Area.

The problem mentioned can be addressed by using electrical sensors at each pole. Each pole is given its unique ID, and if any problem has occurred in an electric pole it has been intimated automatically to the power monitoring stations, and power supply to that electrical pole is stopped to avoid further damage to the poles.

One's problem has been recognized by the sensors it needs to be sent to powerline monitoring stations all this are done through the transmission medium called IOT. IOT (Internet of Things) which allowstwo things to communicate through the internet.

In cloud all the IDs of electrical poles have been stored and in case of any problem in any electrical poles its ID is send to cloud which is pre-stored already and if same ID is matched it stops current flow to that electric pole and transmits information to power monitoring station that this particular pole has been damagedeven using GPS a location can be found, and even standard text message is sent to the in charge person saying that this specific pole has been damaged.

The advantage in our project: In our project every sensors and node units assure exact power quality information and detects if any problem occurs like short circuit etc. and sends the data without any disturbance. 


\section{EXPERIMENTAL RESULTS}

Prototype Model

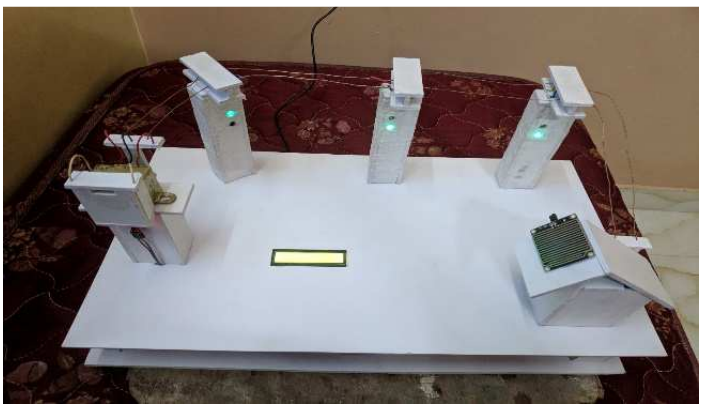

Figure 5

Application Screenshots

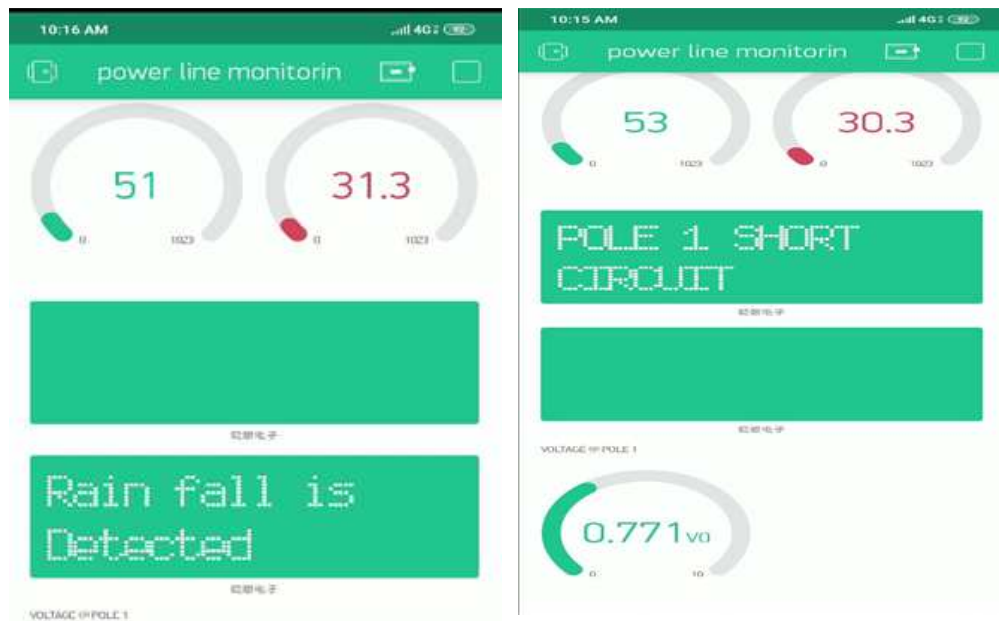

Figure 6
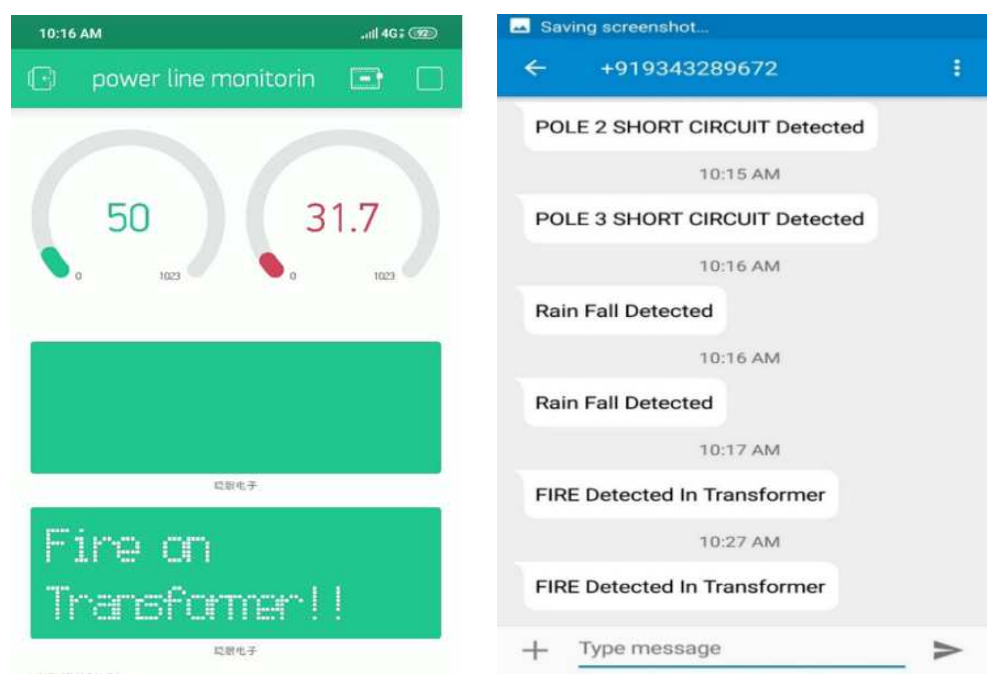

Figure 7 


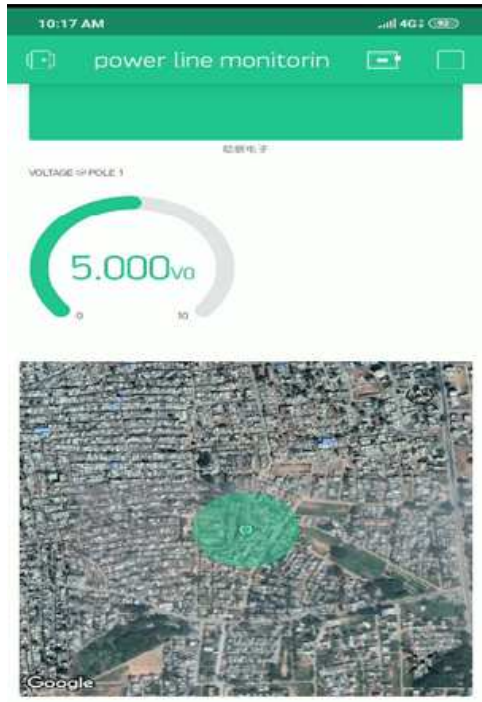

Figure 8

\section{Automated Message sent to the Area Supervisor}

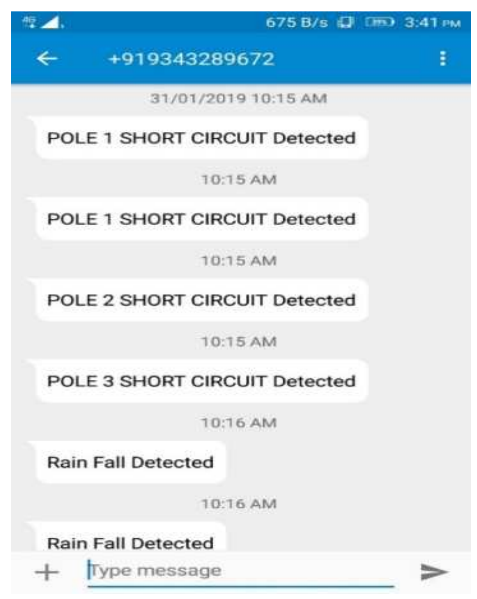

Figure 9

\section{CONCLUSIONS}

Smart Grid (SG) is that the future grid that solves the issues of power variation, and loss of power because of natural calamities, energy wastage, growing energy demand, dependableness and security in the traditional power grid. The Internet of Things (IoT) technology provides connectivity to anyplace at any time. It helps SG by providing smart devices or IoT devices (such as sensors and actuators) for the observance, analysis and dominant the grid, further as property, automation, and pursuit of such devices. This realizes the IoT-aided SG system that supports and improves numerous network functions at the ability generation, transmission, distribution, and utilization. In our project, we've given a comprehensive survey on IoT-aided SG systems. We've surveyed architectures and applications of IoT-aided SG systems. We have a tendency to mention the existing and potential applications of IoT-aided SG systems. We have a tendency to additionally given numerous IoT and non-IoT communication technologies for SG systems by presenting their benefits, disadvantages, and pertinency. 
Additionally, some existing prototypes of IoT-aided SG systems were surveyed which might function a baseline for future analysis during this space. Since IoT-aided SG systems will generate a colossal quantity of information, therefore, we have a tendency to additionally surveyed and provided solutions for important processing in IoT-aided SG systems. We have a tendency to over the survey by presenting open problems, challenges, and future analysis directions for IoT-aided SG systems.

\section{REFERENCES}

1. Ducpyo Hong, Jinmok Lee, Jaeho Choi. "PowerQuality Monitoring System Using Power LineCommunication", 2005 5th InternationalConference on Information Communications \&Signal Processing, 2005.

2. Xi Chen, Limin Sun, Hongsong Zhu, Yan Zhen, Hongbin Chen. "Application of Internet of Things in Power-Line Monitoring", 2012 International Conference on Cyber-Enabled Distributed Computing and Knowledge Discovery, 2012.

3. ZhouYao."Transmission line temperature online monitoring system based on Zig Bee", 2009 International Conference on Sustainable PowerGeneration and Supply, 04/2009.

4. Qing Wang, Yonghua Lin, Hai Zhan. "A hybridwireless system for power line monitoring", IEEE PES Innovative Smart GridTechnologies2012.

5. Natalie matta, Rana Rahim- Amoud, Leila Merghem-Boulahia and Akil "A wireless sensor network for substation monitoring and control in the smart grid." Troyes University of technology September 19-21, 2016, Poland.

6. $\quad$ M Mc Granaphan "Trends in power Quality Monitoring." IEEE Power Eng. Review, pp 3-9,2001

7. Thakur, R. O. B. I. N., Suri, A. R. S., Kumar, S., \& Kumar, A. N. I. L. (2013). A review of integrated renewable energy system in power generation. Int J Mech Prod Eng Res Technol, 3, 79-88.

8. Vehbi C. Gungor, Member, IEEE, Bin Lu, Senior Member "Opportunities and Challenges of Wireless Sensor Networks in Smart Grid" IEEE 2010

9. Celio Fonseca Barbosa e Flávio Eduardo Nallin "Lightning Protection of a Smart Grid Sensor." 